\title{
Pedicled Latissimus Dorsi Flap for Complex Arm Defects: A Report of Twenty Cases from Two Centers
}

\author{
HAZEM ELTAYEB, M.D.*; EMAD SALAH, M.D.*; ASHRAF MOSTAFA, M.D.* and \\ MUNEERA BEN NAKHI, M.D.** \\ The Plastic Surgery Unit, General Surgery Department, Faculty of Medicine, Zagazig University* and \\ Adan Hospital, Ministry of Health, Kuwait**
}

\begin{abstract}
Introduction: The reconstruction of extensive defects of the upper limb are challenging regarding both coverage and function. Latissimus dorsi is a suitably located muscle for resurfacing defects in the arm, elbow and forearm especially with exposure of the vital structures. It is a large flap, with a long pedicle and can be used as a muscle or musculocutaneous flap.
\end{abstract}

Aim of the Work: Evaluate the role of pedicled latissimus dorsi flap in reconstruction of complex posttraumatic arm defects.

Patients and Methods: Pedicled Latissimus dorsi flaps with split thickness skin graft were used to reconstruct 20 arm defects. The study were performed in two plastic surgery units in Egypt and Kuwait. Overall flap survival and postoperative complications were used as outcome measures.

Results: None of our flap was totally necrosed. Two cases $(10 \%)$ developed partial necrosis and partial graft loss occurred in 2 cases $(10 \%)$. Donor site seroma formation was seen in 4 cases $(20 \%)$. Four cases developed stretching of the scar. Followup ranged from (6-24 months), in which Latissimus dorsi healed perfectly, atrophied and contoured well with a cosmetically satisfactory result. No functional deficit developed from the loss of the latissimus dorsi.

Conclusion: Pedicled latissimus dorsi flap is reliable, versatile, and could be the primary choice for reconstruction of post traumatic large complex arm defects.

Key Words: Soft tissue defects - Arm - Latissimus dorsi muscle-Pedicled flap.

\section{INTRODUCTION}

Extensive soft tissue defects of the upper limb are challenging to reconstruct regarding both coverage and function. These defects may result from trauma, tumour excision, infections and radiations. Various reconstructive options ranging from skin grafts, local flaps, distant pedicle flaps and free flaps are available [1].
Local flaps can be used for smaller defects providing skin coverage with similar tissue texture. However, they are limited in size and availability of tissue and also add an injury on already traumatized limb [2]. For any moderate to extensive defect of upper extremity especially with exposed bone, nerves and joints, distant flap either pedicled or free flap is a suitable option [3].

The latissimus dorsi muscle (LDM) flap is an extraordinary and versatile flap that has been a workhorse in the reconstructive surgery for decades [4]. It has a long reliable pedicle with large diameter and despite its large size, no practical functional motor deficit results from its transposition [5].

Depending on its main vascular pedicle, it could be used as a pedicled flap for breast reconstruction, resurfacing defects of anterior chest wall, shoulder and upper arm and head and neck defects up to the temporo-parietal area. As a free flap, LDM flap could be used for coverage of almost all body areas i.e. head and neck, upper and lower limbs and torso. It could be used as myo-cutaneous, osseomyo-cutaneous or isolated muscle flap. Sometimes it may be used as a schemeric flap combined with serratus anterior muscle or as a split muscle flap $[5,6]$.

The scope of pedicled latissimus dorsi flap has expanded in reconstruction of the forearm, elbow, upper arm and shoulder defects [4].

In our study, we evaluated using pedicled latissimus dorsi flap for complex arm defects resulting from trauma in two plastic surgery units from Egypt and Kuwait. 


\section{PATIENTS AND METHODS}

Our case series included 20 patients admitted in both Zagazig University Hospital in Egypt, and Adan Hospital in Kuwait from December 2013 to December 2017.

All cases had post traumatic extensive soft tissue defects in the arm reconstructed by pedicled latissmus muscle flap followed by partial thickness skin graft. For each patient, complete history, general physical, local examination, photographic documentation and routine preoperative preparation was done. We recorded mechanism of injury, associated injury, extent of tissue loss and depth of the defect, timing of coverage (Table 1). The outcome in terms of flap survival, graft take, postoperative complications, cosmesis, function, and patient satisfaction at final follow-up was assessed.

Table (1): Sex, types of trauma, associated injuries, exposed vital structures, timing of flap harvesting and type of LD Flap.

\begin{tabular}{lll}
\hline Item & No. & $\%$ \\
\hline Sex: & 16 & 80 \\
$\quad$ Male & 4 & 20 \\
$\quad$ Female & & \\
Type of trauma: & 14 & 70 \\
$\quad$ RTA & 6 & 30 \\
$\quad$ Machinery & & \\
Associated injury: & 12 & 60 \\
$\quad$ Neurovascular & 10 & 50 \\
$\quad$ Humerus fracture & & \\
Exposed vital structures: & 4 & 20 \\
$\quad$ Bone \& Joint & 8 & 40 \\
$\quad$ Neurovascular & & \\
Timing of flap harvesting: & & 40 \\
$\quad$ Immediate & 12 & 60 \\
$\quad$ Delayed & & \\
Type of LD Flap: & 16 & 80 \\
$\quad$ Muscle & 4 & 20 \\
$\quad$ Musculocutaneous & & \\
$\quad$ & &
\end{tabular}

The ages of patients ranged from 22 to 55 years (mean 28.5 years). Of these 20 cases, 4 were females and 16 were males. In 12 patients, the cause of defect was a road traffic accident (RTA) and in 8 , the cause was industrial machinery injury. Neurovascular structures injury were documented in 12 cases with exposure after repair in 8 cases. Fracture humerus was encountered in 10 cases with exposed bone in 4 cases. Eight cases were managed immediately (all cases associated with neurovascular exposure) where immediate flap harvesting was performed after wound debridement and stabilization of fractures and vascular and nerve repair (Fig. 1A-1E). The rest 12 cases were managed late where debridement was needed as a first step then after 3-7 days, flap harvesting was done (Fig. 2A-2E).

In 16 cases, the LD was harvested as muscle flap while in 4 cases skin paddle was added. Coverage of the flap with split thickness skin graft (STSG) was done 7 days later to be sure that the flap was healed and permitted sound graft take (Figs. 1C, 2D).

Approval for this study was obtained from our institutional review boards. All the cases were done by at least one of authours, and the patients were followed-up for a period of 6 months to 2-year.

\section{Technique of flap harvesting:}

Under general anaesthesia, the patients were placed in a lateral position. The arm and lateral chest and back are prepared and draped. The length of muscle flap required was determined by the distance of latissimus dorsi at insertion to humerus to the distal most part of the wound. The flap width is determined by the width of the defect.

The incision began at the posterior aspect of axilla and passed along the lateral border of the latissimus dorsi toward the iliac crest. Wide undermining of skin was done for easy identification of surrounding muscle anatomy. The anterior border of the latissimus dorsi was identified. During muscle dissection, care was taken to separate the muscle from the superior surface of the scapula and its associated serratus anterior muscle. Whole latissimus dorsi muscle down to the iliac crest was raised and released. Intercostals and para-spinal perforators were carefully ligated. The neurovascular pedicle was identified more proximally on the anterior surface of the muscle and carefully dissected. The branch to the serratus anterior is carefully ligated, and the pedicle is dissected to the circumflex scapular arterial branch to increase the arc of rotation. The tendinous insertion was left intact to guard against excessive traction on the pedicle. Flap was brought to the arm defect without subcutaneous tunnel and after insetting the flap, suction drain was placed in the recipient area. In all cases, the donor site was closed primarily on suction drain. The extremity was elevated postoperatively and was immobilized for total 4 weeks then gradual mobilization started. 


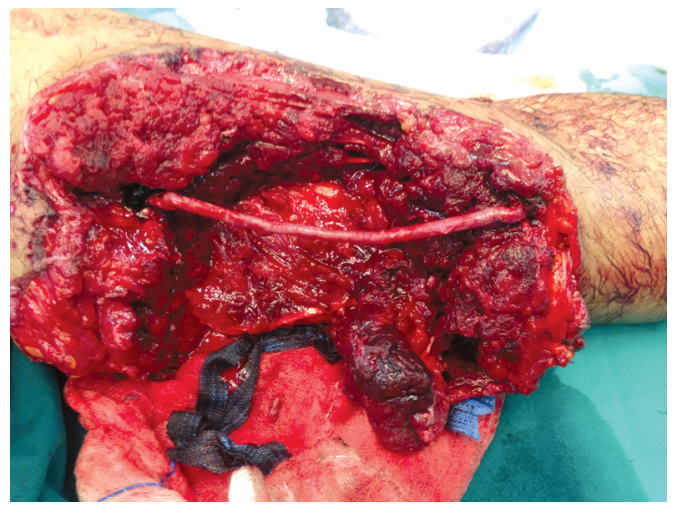

Fig. (1A): Intraoperative picture showing extent of soft tissue loss and exposed venous graft.

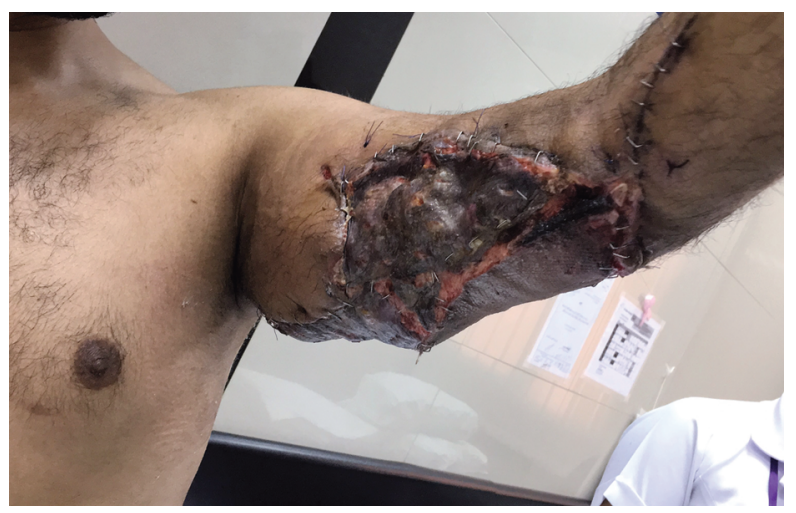

Fig. (1C): Skin graft was done 7 days later to cover remaining exposed LD muscle.

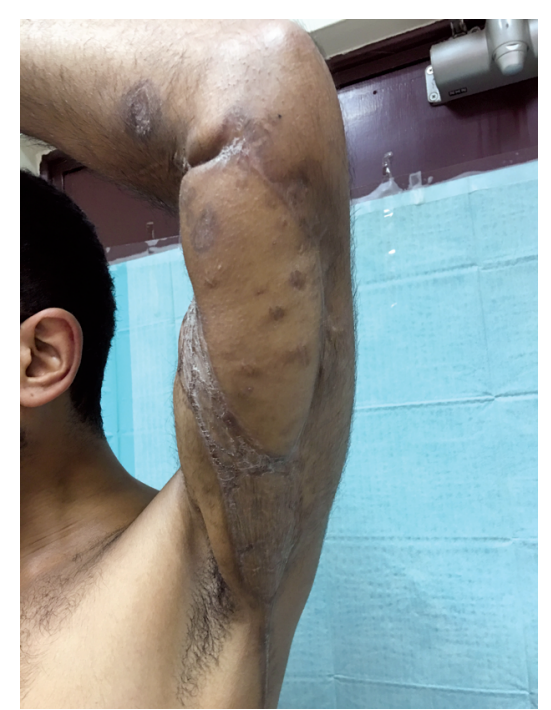

Fig. (1E): Maintained shoulder function after LD flap harvesting.

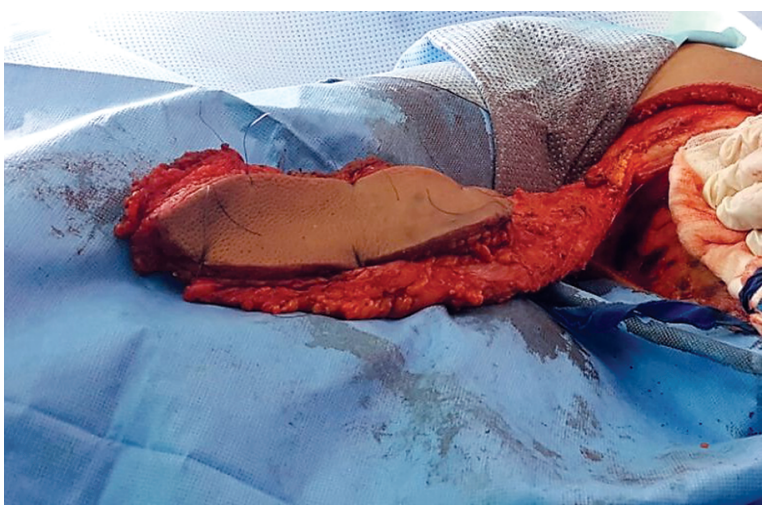

Fig. (1B): The LD flap raised as a myocutaneous flap.

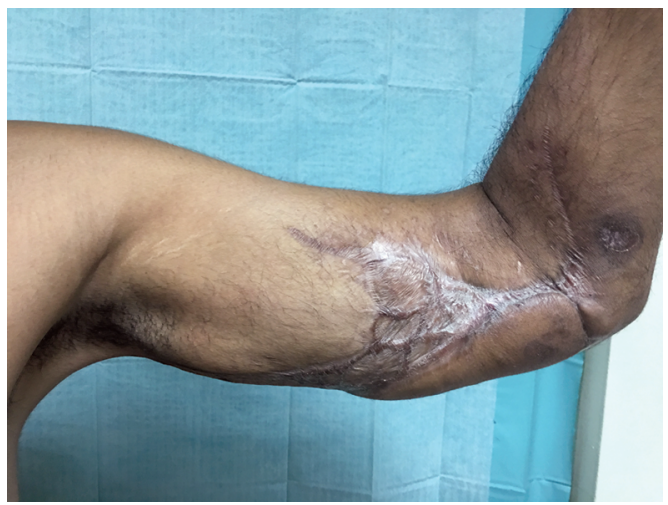

Fig. (1D): Follow-up (18 months); the muscle atrophied and contour well.

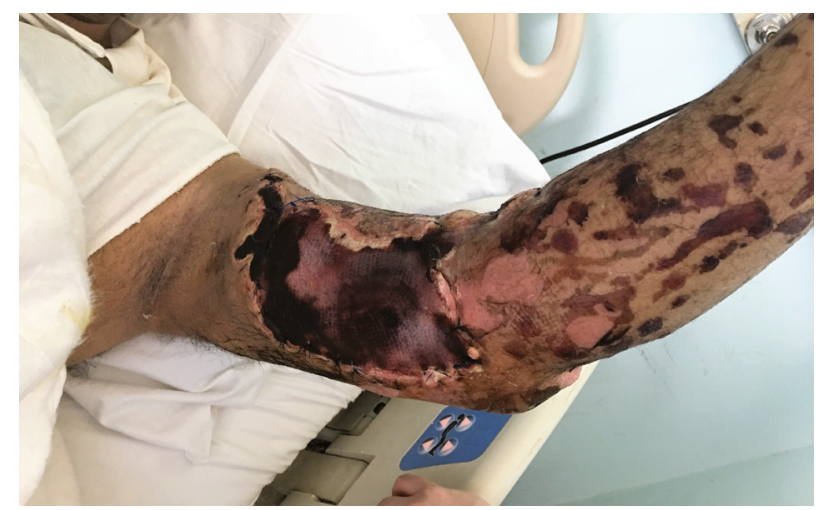

Fig. (2A): Case presented late with soft tissue gangrene. 


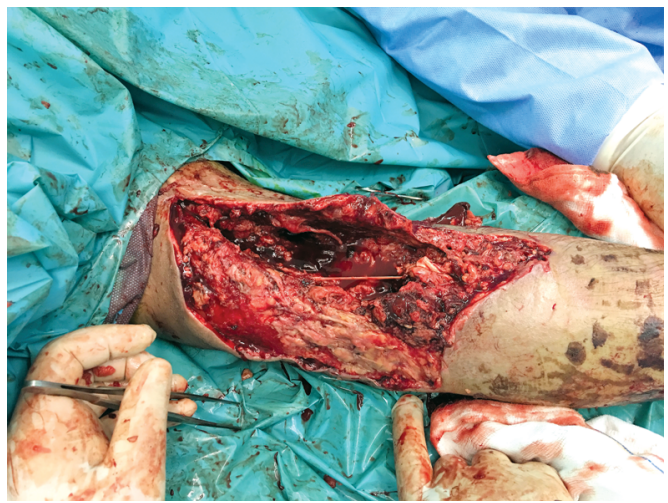

Fig. (2B): After debridement showing loss of flexor muscles and exposed repaired brachial artrery.

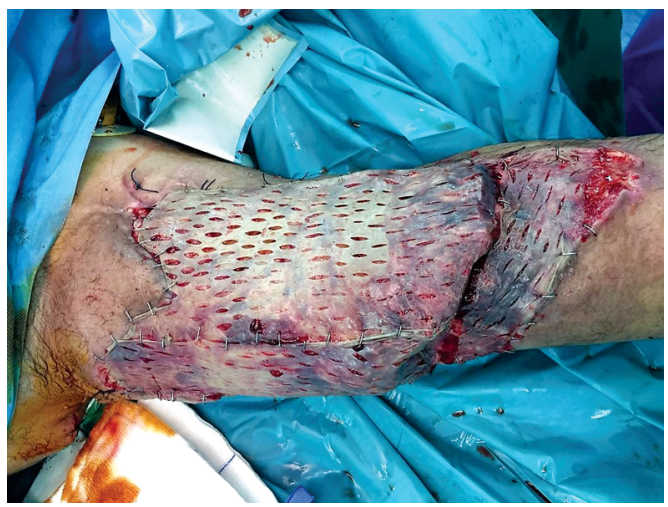

Fig. (2D): Skin graft was done one week later.

\section{RESULTS}

Overall flap survival and postoperative complications were used as outcome measures (Table 2). Eighteen flaps (90\%) survived completely. However 2 cases $(10 \%)$ developed partial distal necrosis. Debridement was done and the wound healed with secondary intention. Other minor complications were partial skin graft loss seen in 2 cases $(10 \%)$ and wound infection seen in 2 cases $(10 \%)$, who were treated successfully with conservative management. In all cases reconstructive goal was achieved at the end. The donor defect was closed primarily in all cases. Donor site seroma formation was seen in 4 cases (20\%) which was resolved with serial needle aspiration. Four cases $(20 \%)$ developed stretching of the scar. There was no scar contracture seen at the donor site in any patient. Mean duration of followup was 9 months (ranged from 6 to 24 months). Latissimus dorsi healed perfectly, atrophied and contoured well to the defects. All patients attained a cosmetically satisfactory result. No functional deficit developed from the loss of the latissimus dorsi.

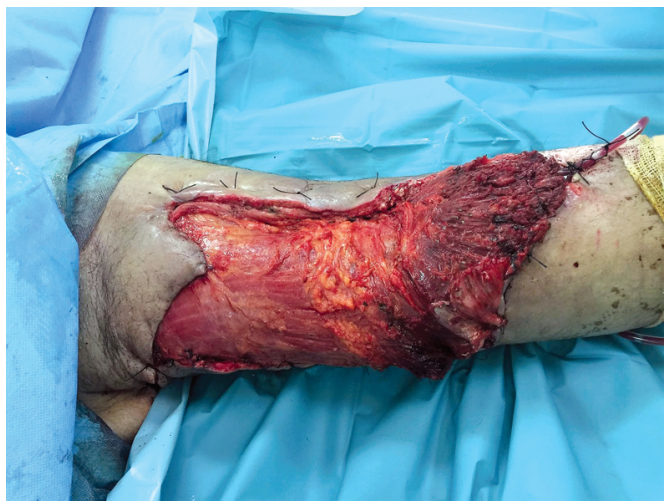

Fig. (2C): The LD flap was raised as muscle flap.

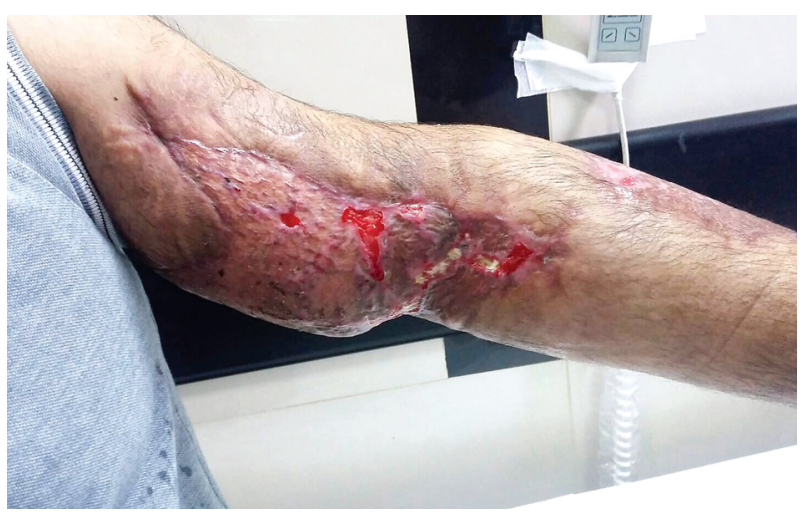

Fig. (2E): One month post operative with good muscle setting and good graft take.

Table (2): Flap and donor site morbidity.

\begin{tabular}{lll}
\hline Item & No. & $\%$ \\
\hline Flap morbidity: & & \\
Total necrosis & 0 & 0 \\
Partial necrosis & 2 & 10 \\
$\quad$ Graft loss & 2 & 10 \\
Infection & 2 & 10 \\
Donor-site morbidity: & & \\
$\quad$ Seroma & 4 & 20 \\
$\quad$ Wound stretching & 4 & 20 \\
\hline
\end{tabular}

\section{DISCUSSION}

The reconstruction of extensive defects of the upper limb are challenging for plastic surgeons to salvage a limb or at least restore its function to the least needed in daily activities after receiving a mutilating trauma [6]. For such extensive defects especially with exposure of the vital structures, pedicled latissimus dorsi flap is a reliable and versatile option $[6,7]$.

Latissimus dorsi is a suitably located muscle for resurfacing defects in the arm, elbow and 
forearm. It is a large flap, with a long pedicle and can be used as a muscle or musculocutaneous flap. A well vascularized flap improves circulation with increased resistance to infection and promotes fracture union $[4,8]$.

In this study we had resurfaced twenty posttraumatic complex arm defects using LDM flaps followed by partial thickness skin graft. The study was conducted in 2 plastic surgery units in Egypt and Kuwait. None of our flap was totally necrosed, however, we faced minor flap and graft morbidity that were treated successfully with conservative wound management. Donor site morbidity included scar stretching and seroma formation. Our results are near to previous reports from our medical institution in Egypt [5,6]. In The first report, seventeen flaps were harvested to reconstruct mutilating upper limb injuries in pediatric group. None of them was lost. Split thickness skin graft was done one week after flap harvesting and insetting to cover the muscle flap. There were four cases with partial loss of the skin graft secondary to infection mandating re-grafting [5].

In the second report, $10 \mathrm{LD}$ flaps were harvested (7 as muscle flaps and 3 as myocutaneous flaps) to reconstruct complex elbow injuries. None of these flaps was lost. Nine flaps $(90 \%)$ survived completely while one case (10\%) developed marginal flap necrosis where it was debrided and grafted. No mortalities were reported in this series. Donor-site morbidity was in the form of seroma collection (2 cases), superficial wound infection ( 2 cases) and partial wound dehiscence (only one patient). None of these donor-site complications needed surgical intervention [6].

Also, we are comparable to the work presented by Mohanty and Nayak [4] in their report of 23 cases. They reconstructed defects resulting from trauma (19 cases) and neoplastic resection (4 cases). Patients' ages ranged of 15-45 years. The average duration of follow-up was 6 months to 1 year. All the flaps survived without partial loss. Six patients developed seroma at flap donor site which settled with multiple aspirations. Two patient developed hypertrophy at the borders at the donor site scar. Three patients had stretching of the scar. All patients have cosmetically satisfactory result. They considered Pedicled Latissimus dorsi muscle and musculocutaneous flap with a split thickness skin graft as the primary choice for reconstruction of large complex arm defects.

Sajjad et al. [2] in another report used pedicled latissimus dorsi flap for reconstruction of extensive defects around the elbow with $90 \%$ success rate and the survival of flap was $100 \%$, with partial flap loss (necrosis) in 10\% of patients. They documented, donor site seroma formation in 8 patients $(28 \%)$ and minor functional deficit after muscle harvesting which was overcome by physiotherapy. These results are similar to our study regarding flap survival and donor site morbidity.

Ma et al. [9], adopted the use of pedicled LDM flap for reconstruction of upper extremity large soft-tissue defects. The ages of their patients was ranging between 17-67 years. reported that transfer of the pedicled latissimus dorsi muscle flap is a very useful procedure in the reconstruction of the upper extremity and considered safe, simple and reliable. Their 20 flaps survived without loss. Only minor complications of flap edge necrosis and wound breakdown were found in three patients, and varying degrees of minor split-thickness skin graft loss were present in five patients. No deep infections were found in their series. Those are more or less comparable to our results mentioned in Table (2).

Lai et al. [10] used myocutaneous latissimus dorsi flap for coverage and functional transfer in a burn patient with a large shoulder wound and destroyed deltoid muscle, they recommended tunneling the flap posteriorly to the shoulder to avoid traction on the pedicle Itoh et al. [11] and Ferrier et al. [12] described tunneling the muscle anteriorly to provide coverage and anterior and middle deltoid function. In our study, we avoided tunneling whenever possible to avoid unexpected muscle pedicle compression and found this more safe without affecting the final reconstructive goal.

Stern and Carey [13] and Minami et al. [14] advocated detaching both the origin and the insertion of the muscle in all cases of pedicled flap to increase the mobility of the flap. They have also reported complete loss of the flap due to kinking or twisting of the pedicle during transfer. In the contrary here, the latissimus dorsi tendon was left intact in all our cases, to avoid excessive traction on the vascular pedicle.

Bailey and Godfrey preferred using split thickness skin graft in conjunction with latissimus free flaps for soft tissue coverage because it decreases donor site morbidity and provides cosmetically superior coverage [15]. Stevenson et al. [16] attained good functional and cosmetic results in a 12 years old trauma victim in whom a pedicled flap and a meshed split thickness skin graft had been used 
for soft tissue coverage and to restore function. We agree with that result and we have used muscle flap with SSG in all cases in our series, even in the four cases where skin paddle was added. We choose the muscle with minimally meshed skin graft because it is less bulky. The muscle contours well as it atrophies and produces excellent cosmesis.

We used latissimus dorsi flap in arm with exposed humerus with underlying fracture, exposed neurovascular bundle, exposed shoulder joint, osteomyelitis and posttraumatic loss of flexor group requiring cover of interposition vein graft following segmental loss of brachial artery and restoration of elbow flexion.

Microvascular free tissue transfer can be a good choice in difficult situations but in heavily traumatized limbs a healthy recipient vessel can be a major problem. Free tissue transfer is also not without limitations. It needs expert and skilled surgeons, anesthesia, nursing staff, special equipments and instruments and fit patient for a long surgery. Postoperative vascular complication may result in flap failure and donor site morbidity as well $[\mathbf{5 , 8}]$. For that, we preferred pedicled flap to free flap because the procedure is quicker one, safe, and technically less challenging especially in state of emergency.

\section{Conclusion:}

Pedicled latissimus dorsi flap is reliable, versatile, quick to execute and has a wide arc of rotation. We recommend it as the primary choice for reconstruction of post traumatic large complex arm defects. Donor site morbidity is minimal and functional and cosmetic outcome are very accepted.

\section{REFERENCES}

1- Tan O., Atik B. and Ergen D.: Versatile use of the pedicled latissimus dorsi flap as a salvage procedure in reconstruction of complex injuries of the upper extremity. Ann. Plast. Surg., 59: 501-506, 2007.

2- Sajjad Y., Hameed A., Gill N.A. and Bhutto A.W.: Use of A Pedicled Flap for Reconstruction of Extensive Soft Tissue Defects Around Elbow. J. Coll. Physicians Surg. Pak., 20 (1): 47-50, 2010.

3- Herter F. and Ninkovic M.: Rotational flap selection and timing for coverage of complex upper extremity trauma. J. Plastic. Reconstr. Aesthet. Surg., 60: 760768, 2007.

4- Mohanty N. and Nayak B.B.: Pedicled latissimus dorsi flap for arm defects: A report of twenty-three cases. J. Orthop. Traumatol. Rehabil., 7: 145-149, 2014.

5- Abo-Hashem A. and Zakaria Y.: Role of Latissimus Dorsi Island Flap in Coverage of Mutilating Upper Limb Injuries in Pediatric Age Group. Annals of Pediatric Surgery., 6 (3): 154-160, 2010.

6- Nasr M., Abo-Hashem A. and Anany R.: Role of Denervated Latissimus Dorsi Island Flap in Reconstruction of Complex Elbow Injuries. Egypt, J. Plast. Reconstr. Surg., 36 (2): 209-213, 2012.

7- Pierce T.D. and Tomaino M.M.: Use of the pedicled latissimus muscle flap for upper extremity reconstruction. J. Am. Acad. Orthop. Surg., 8: 324-331, 2000.

8- Mordick T.G., Britton E.N. and Brantigan C.: Pedicled latissimus dorsi transfer for immediate soft-tissue coverage and elbow flexion. Plast. Reconstr. Surg., 99: 1742-1744, 1997.

9- Ma C.H., Tu U.K., Wu C.H., et al.: Reconstruction of upper extremity large soft tissue defects using latissimus dorsi muscle flap-technique illustration and clinical outcomes. Injury, 39 (Oct; Suppl.), 4: 67-74, 2008.

10- Lai M.F., Milroy B.C. and Pennington D.G.: Shoulder defect cover with functional restoration using the latissimus dorsi myocutaneous flap: A case report. Br. J. Plast. Surg., 35: 140-143, 1982.

11- Itoh Y., Sasaki T., Ishiguro T., Uchinishi K., Yabe Y. and Fukuda H.: Transfer of latissimus dorsi to replace a paralysed anterior deltoid. A new technique using an inverted pedicled graft. J. Bone Joint Surg. Br., 69: 647$751,1987$.

12- Ferrier J.A., Owens T. and Singer D.I.: Functional reconstruction of the shoulder with a latissimus dorsi pedicle flap and a hemiarthroplasty: A case report. J. Hand Surg. Am., 20: 284-287, 1995.

13- Stern P.J. and Carey J.P.: The latissimus dorsi flap for reconstruction of the brachium and shoulder. J. Bone Joint Surg. Am., 70: 526-535, 1988.

14- Minami A., Ogino T., Ohnishi N. and Itoga H.: The Latissimus dorsi musculocutaneous flap for extremity reconstruction in orthopedic surgery. Clin. Orthop., 260: 201-206, 1990.

15- Bailey B.N. and Godfrey A.M.: Latissimus dorsi muscle free flaps. Br. J. Plast. Surg., 35: 47-52, 1982.

16- Stevenson T.R., Duus E.C., Greene T.L. and Dingman R.O.: Traumatic upper arm defect treated with latissimus dorsi muscle transposition. J. Pediatr. Orthop., 4: 111113, 1984. 